\title{
EVOLUTES OF HYPERBOLIC DUAL SPHERICAL CURVE IN DUAL LORENTZIAN 3-SPACE
}

\author{
RASHAD A. ABDEL-BAKY ${ }^{1,2, *}$
}

\begin{abstract}
Based on the E. Study's map, we study a timelike ruled surface as a curve on the hyperbolic dual unit sphere in dual Lorentzian 3 -space $\mathbb{D}_{1}^{3}$. Then, as applications of the singularity theory of smooth functions, we define the notation of evolutes for timelike ruled surfaces and establish the relationships between their geometric invariants. Finally, an example of application is introduced and explained in detail.
\end{abstract}

\section{INTRODUCTION}

Despite its long history, the theory of surface is still one of the most important interesting topics in differential geometry and it is being study by many mathematicians until now. Among the surfaces, a ruled surface has been drawing attention to scientists as well as mathematicians because of its various application such as the study of design problems in spatial mechanisms and physics, kinematics and computer aided design (CAD). There exists a vast literature on the subject including several monographs, for example [1-7].

Rather unexpectedly dual numbers have been applied to study the motion of a line space; they seem even be the most appropriate apparatus for this end. In screw and dual number algebra, the E. Study's map concludes: The set of all oriented lines in Euclidean 3 -space $\mathrm{E}^{3}$ is in one-to- one correspondence with set of points of the dual unit sphere in the dual 3- space $\mathbb{D}^{3}$. More details on the necessary basic concepts about the dual elements and the one-to-one correspondence between ruled surfaces and one-parameter dual spherical motions can be found in [7-10]. If we take the Minkowski 3 -space $\mathbb{E}_{1}^{3}$ instead of $\mathrm{E}^{3}$ the E. Study's map can be stated as follows: The timelike and spacelike dual unit vectors of hyperbolic and Lorentzian dual unit spheres $\mathbb{H}_{+}^{2}$ and $\mathbb{S}_{1}^{2}$ at the Lorentzian 3-space $\mathbb{D}_{1}^{3}$ are in one-to-one correspondence with the directed timelike and spacelike lines of the space of Lorentzian lines $\mathbb{E}_{1}^{3}$, respectively [12]. Then a differentiable curve on $\mathbb{H}_{+}^{2}$ corresponds to a timelike ruled surface at $\mathbb{E}_{1}^{3}$. Similarly the timelike (resp. spacelike) curve on $\mathbb{S}_{1}^{2}$ corresponds to any spacelike (resp. timelike) ruled surface at $\mathbb{E}_{1}^{3}$. Then, the study of ruled surfaces in the Minkowski 3-space is more interesting than the Euclidean case.

One of the main techniques for applying the singularity theory to Euclidean differential geometry is to consider the distance squared function and the height function on a submanifold of $\mathrm{E}^{3}[13,14]$. There are some articles concerning singularities of surfaces and classical geometric invariants of space curves for several kinds of geometry [13-23]. In these articles the corresponding functions depend on each geometry. In this paper, we consider the Lorentzian dual distance function on a dual curve in $\mathbb{H}_{+}^{2}$. As an application of singularity theory to the Lorentzian dual height function, we detect the hyperbolic dual evolute and classify singularities of it. By the main result we showed that the hyperbolic dual evolute can be defined in the case when the dual geodesic curvature $\Sigma \neq \pm 1$. Then, applying to Study's map, we established the relationships between singularities of these subjects and geometric invariants of timelike ruled surface which are deeply related to the order of contact with evolutes. Finally, an example illustrates the application of the obtained formulae was introduced.

Received $20^{\text {th }}$ September, 2017; accepted $21^{\text {st }}$ October, 2017; published $1^{\text {st }}$ November, 2017.

2010 Mathematics Subject Classification. 53A04, 53A05, 53A17.

Key words and phrases. keyword1; Blaschke frame; evolute of the dual spherical curve; singularity.

(C) 2017 Authors retain the copyrights of their papers, and all open access articles are distributed under the terms of the Creative Commons Attribution License. 


\section{Basic concepts}

In this section we list some notions, formulas and conclusions for the theory of dual numbers and dual Lorentzian vectors (See for instance Refs. [1-4, 8-11]). Let $\mathbb{R}^{3}$ denote the vector space with its usual vector structure. We denote $\left(x_{1}, x_{2}, x_{3}\right)$ the coordinates of a vector with respect to the canonical basis of $\mathbb{R}^{3}$. The three-dimensional Minkowski 3-space is the metric space $\mathbb{E}_{1}^{3}=\left(\mathbb{R}^{3},<,>\right)$, where the metric $<,>$ is

$$
<\mathbf{x}, \mathbf{y}>=-x_{1} y_{1}+x_{2} y_{2}+x_{3} y_{3}, \mathbf{x}=\left(x_{1}, x_{2}, x_{3}\right), \mathbf{y}=\left(y_{1}, y_{2}, y_{3}\right),
$$

which is called the Lorentzian metric. For any two vectors $\mathbf{x}=\left(x_{1}, x_{2}, x_{3}\right)$ and $\mathbf{y}=\left(y_{1}, y_{2}, y_{3}\right)$ of $\mathbb{E}_{1}^{3}$, the Lorentzian vector product is defined by

$$
\mathbf{x} \times \mathbf{y}=\left(-\left(x_{2} y_{3}-x_{3} y_{2}\right),\left(x_{1} y_{3}-x_{3} y_{1}\right),\left(x_{1} y_{2}-x_{2} y_{1}\right)\right) .
$$

A vector $\mathbf{x} \in \mathbb{E}_{1}^{3}$ is said to be spacelike if $\langle\mathbf{x}, \mathbf{x}>>0$ or $\mathbf{x}=\mathbf{0}$, timelike if $\langle\mathbf{x}, \mathbf{x}><0$ and lightlike or null if $\left\langle\mathbf{x}, \mathbf{x}>=0\right.$ and $\mathbf{x} \neq \mathbf{0}$. A timelike or light-like vector in $\mathbb{E}_{1}^{3}$ is said to be causal. We point out that the null vector $\mathbf{x}=\mathbf{0}$ is considered of spacelike type although it satisfies $\langle\mathbf{x}, \mathbf{x}\rangle=0$. For $\mathbf{x} \in \mathbb{E}_{1}^{3}$ the norm is defined by $\|\mathbf{x}\|=\sqrt{|\langle\mathbf{x}, \mathbf{x}\rangle|}$, then the vector $\mathbf{x}$ is called a spacelike unit vector if $\langle\mathbf{x}, \mathbf{x}\rangle=1$ and a timelike unit vector if $\langle\mathbf{x}, \mathbf{x}\rangle=-1$. Similarly, a regular curve in $\mathbb{E}_{1}^{3}$ can locally be spacelike, timelike or null (lightlike), if all of its velocity vectors are spacelike, timelike or null (lightlike), respectively.

The angle between two vectors in Minkowski 3-space $\mathbb{E}_{1}^{3}$ is defined by [8-11]:

Definition 1 i) Spacelike angle: Let $\mathbf{x}$ and $\mathbf{y}$ be spacelike vectors in $\mathbb{E}_{1}^{3}$ that span a spacelike vector subspace; then we have $|<\mathbf{x}, \mathbf{y}\rangle \mid \leq\|\mathbf{x}\|\|\mathbf{y}\|$, and hence, there is a unique real number $\theta \geq 0$ such that $\langle\mathbf{x}, \mathbf{y}\rangle=\|\mathbf{x}\|\|\mathbf{y}\| \cos \theta$. This number is called the spacelike angle between the vectors $\mathbf{x}$ and $\mathbf{y}$. ii) Central angle: Let $\mathbf{x}$ and $\mathbf{y}$ be spacelike vectors in $\mathbb{E}_{1}^{3}$ that span a timelike vector subspace; then we have $|\langle\mathbf{x}, \mathbf{y}\rangle|>\|\mathbf{x}\|\|\mathbf{y}\|$, and hence, there is a unique real number $\theta \geq 0$ such that $<\mathbf{x}, \mathbf{y}\rangle=\|\mathbf{x}\|\|\mathbf{y}\| \cosh \theta$. This number is called the central angle between the vectors $\mathbf{x}$ and $\mathbf{y}$.

iii) Lorentzian timelike angle: Let $\mathbf{x}$ be spacelike vector and $\mathbf{y}$ be timelike vector in $\mathbb{E}_{1}^{3}$. Then there is a unique real number $\theta \geq 0$ such that $\langle\mathbf{x}, \mathbf{y}\rangle=\|\mathbf{x}\|\|\mathbf{y}\| \sinh \theta$. This number is called the Lorentzian timelike angle between the vectors $\mathbf{x}$ and $\mathbf{y}$.

A ruled surface $M$ in $\mathbb{E}_{1}^{3}$ is a surface generated by a straight line $L$ moving along a curve $\mathbf{C}(s)$. The various positions of the generating lines are called the rulings of the surface. Such a surface, thus, has a parametrization in the ruled form [1-6]:

$$
M: \mathbf{y}(s, v)=\mathbf{C}(s)+v \mathbf{x}(s), s \in I, v \in \mathbb{R},
$$

such that $\left\langle\mathbf{x}, \mathbf{x}>=\sigma( \pm 1),\left\langle\mathbf{x}^{\prime}, \mathbf{x}^{\prime}\right\rangle=\eta( \pm 1),\left\langle\mathbf{C}^{\prime}, \mathbf{x}^{\prime}\right\rangle=0 ; \prime=\frac{d}{d s}\right.$. In this case the curve $\mathbf{C}=\mathbf{C}(s)$ is the striction curve, and the parameter $s$ is the arc length of the non-null spherical curve $\mathbf{x}=\mathbf{x}(s)$. Let now excluding $\mathbf{x}$ is constant or null or $\mathbf{x}^{\prime}$ null. As usual Blaschke frame relative to $\mathbf{x}(s)$ will be defined as the frame of which this line and the central normal $\mathbf{t}(s)=\mathbf{x}^{\prime}(s)$ to the ruled surface at the central point are two edges. The third edge $\mathbf{g}(s)=\mathbf{x} \times \mathbf{t}$ is the central tangent to the ruled surface $M$. The frame $\left\{\mathbf{x}=\mathbf{x}(s), \mathbf{t}(s)=\mathbf{x}^{\prime}, \mathbf{g}(s)=\mathbf{x} \times \mathbf{t}\right\}$ is called Blaschke frame. Then, we have

$$
\mathbf{x} \times \mathbf{t}=\mathbf{g}, \quad \mathbf{x} \times \mathbf{g}=\sigma \mathbf{t}, \quad \mathbf{t} \times \mathbf{g}=-\eta \mathbf{x}, \quad<\mathbf{g}, \mathbf{g}>=-\sigma \eta .
$$

Therefore, the following Blaschke formulae hold:

$$
\left(\begin{array}{l}
\mathbf{x}^{\prime} \\
\mathbf{t}^{\prime} \\
\mathbf{g}^{\prime}
\end{array}\right)=\left(\begin{array}{lll}
0 & 1 & 0 \\
-\sigma \eta & 0 & \gamma \\
0 & \sigma \gamma & 0
\end{array}\right)\left(\begin{array}{l}
\mathbf{x} \\
\mathbf{t} \\
\mathbf{g}
\end{array}\right),
$$

where $\gamma(s)=\operatorname{det}\left(\mathbf{x}^{\prime \prime}, \mathbf{x}^{\prime}, \mathbf{x}\right)$ is the geodesic curvature function of the non-null spherical $\mathbf{x}(s)$. In terms of the Blaschke frame $\{\mathbf{x}, \mathbf{t}, \mathbf{g}\}$ with signs $\sigma, \eta,-\sigma \eta$, the striction curve $\mathbf{C}$ can be reconstructed from

$$
\mathbf{C}^{\prime}(s)=\sigma \Gamma \mathbf{x}-\sigma \eta \mu \mathbf{g} .
$$


The functions $\gamma(s), \Gamma(s)$ and $\mu(s)$ are called the curvature functions or construction parameters of the ruled surface. The geometrical meanings of these invariants are explained as follows: $\gamma$ is the geodesic curvature of the spherical image curve $\mathbf{x}=\mathbf{x}(s) ; \Gamma$ describes the angle between the tangent of the striction curve and the ruling of the surface; and $\mu$ is the distribution parameter of the ruled surface at the ruling $\mathbf{x}$. Note that $M$ is a timelike surface when $(\sigma, \eta)=( \pm 1,1)$. In fact $(\sigma, \eta)=(-1,-1)$ is impossible because of the causal character.

2.1. The E. Study's map. The set of dual numbers is

$$
\mathbb{D}=\left\{A=a+\varepsilon a^{*} \mid a, a^{*} \in \mathbb{R},\right.
$$

where $\varepsilon \neq 0$ is called the dual operator with the algebraic property of $\varepsilon^{2}=0$. Sums and products of dual numbers are well defined using the dual operator. Analogously, for all pairs $\left(\mathbf{x}, \mathbf{x}^{*}\right) \in \mathbb{E}_{1}^{3} \times \mathbb{E}_{1}^{3}$ the set

$$
\mathbb{D}_{1}^{3}=\left\{\mathbf{X}=\mathbf{x}+\varepsilon \mathbf{X}^{*}, \varepsilon \neq 0, \varepsilon^{2}=0\right\},
$$

together with the Lorentzian inner product

$$
<\mathbf{X}, \mathbf{Y}>=<\mathbf{x}, \mathbf{y}>+\varepsilon\left(<\mathbf{y}, \mathbf{x}^{*}>+<\mathbf{y}^{*}, \mathbf{x}>\right)
$$

forms the dual Lorentzian 3 -space $\mathbb{D}_{1}^{3}$. Thereby a point $\mathbf{X}=\left(X_{1}, X_{2}, X_{3}\right)^{t}$ has dual coordinates $X_{i}=\left(x_{i}+\varepsilon x_{i}^{*}\right) \in \mathbb{D}$. The norm is defined by

$$
<\mathbf{X}, \mathbf{X}>^{\frac{1}{2}}:=\|\mathbf{X}\|=\|\mathbf{x}\|\left(1+\varepsilon \frac{<\mathbf{x}, \mathbf{x}^{*}>}{\|\mathbf{x}\|^{2}}\right) .
$$

The hyperbolic and Lorentzian dual unit spheres, respectively, are:

$$
\mathbb{H}_{+}^{2}=\left\{\mathbf{X} \in \mathbb{D}_{1}^{3} \mid-X_{1}^{2}+X_{2}^{2}+X_{3}^{2}=-1, X_{1}>0\right\},
$$

and

This yields

$$
\mathbb{S}_{1}^{2}=\left\{\mathbf{X} \in \mathbb{D}_{1}^{3} \mid-X_{1}^{2}+X_{2}^{2}+X_{3}^{2}=1\right\}
$$

$$
\mathbf{F}_{1} \times \mathbf{F}_{2}=\mathbf{F}_{3}, \mathbf{F}_{2} \times \mathbf{F}_{3}=-\mathbf{F}_{1}, \mathbf{F}_{3} \times \mathbf{F}_{1}=\mathbf{F}_{2},
$$

where $\mathbf{F}_{1}, \mathbf{F}_{2}$, and $\mathbf{F}_{3}$, are the base at the origin point $\mathbf{0}(0,0,0)$ of the dual Lorentzian 3 -space $\mathbb{D}_{1}^{3}$. Via this, the E. Study's map can be stated as follows: The dual unit spheres are shaped as a pair of conjugate hyperboloids. The common asymptotic cone represents the set of null lines, the ring shaped hyperboloid represents the set of spacelike lines, and the oval shaped hyperboloid forms the set of timelike lines, opposite points of each hyperboloid represent the pair of opposite vectors on a line (see Fig. 1).

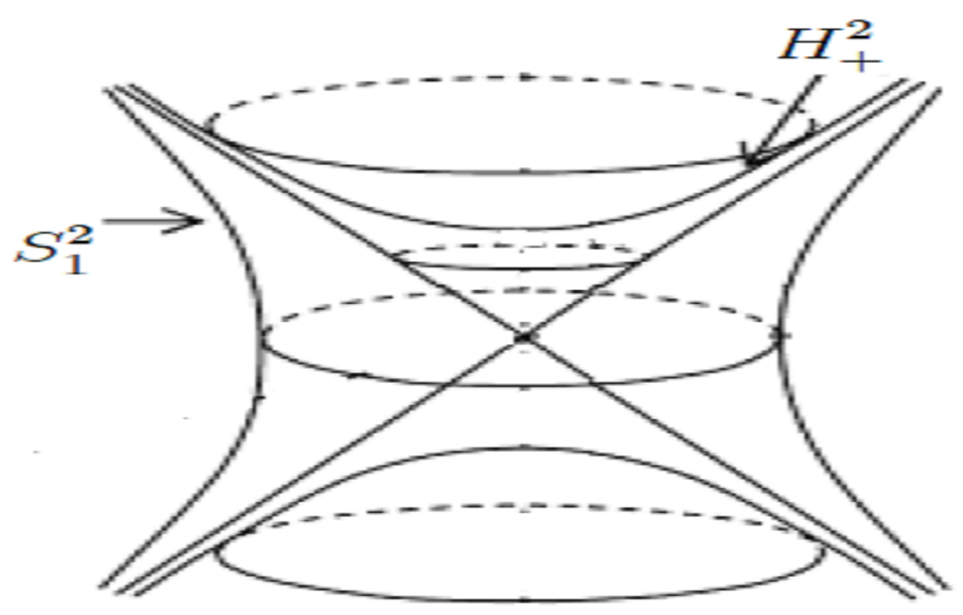

Figure 1 


\section{Timelike Ruled surface as a DUAL CURVE}

We use in this section the notations of the preceding section. The E. Study's map allows us to rewrite Eq. (2.3) by the dual vector function as:

$$
M: \mathbf{X}(s)=\mathbf{x}(s)+\varepsilon \mathbf{C}(s) \times \mathbf{x}(s)=\mathbf{x}(s)+\varepsilon \mathbf{x}^{*}(s),
$$

where $\mathbf{x}^{*}$ is the moment of $\mathbf{x}$ about the origin in $\mathbb{E}_{1}^{3}$. The representation of directed lines in $\mathbb{E}_{1}^{3}$ by dual unit vectors brings about several advantages and from now on we do not distinguish between directed lines and their representing dual unit vectors. Therefore, the dual arc length $d S=d s+\varepsilon d s^{*}$ of the dual curve $\mathbf{X}(s) \in \mathbb{H}_{+}^{2}$ or $\mathbb{S}_{1}^{2}$ is:

$$
S(s)=\int_{s_{0}}^{s} \sqrt{\left|<\mathbf{X}^{\prime}, \mathbf{X}^{\prime}>\right|} d s
$$

Then the dual parameter is determined such that $\left\|\frac{d \mathbf{X}}{d S}\right\|=1$. So, we have

$$
\frac{d s}{d S}=\frac{1}{\left|<\mathbf{X}^{\prime}, \mathbf{X}^{\prime}\right\rangle \mid} \text {. }
$$

Therefore, we derive the dual Lorentzian form of the Blaschke frame equations in exactly the same way as in Eq. (2.5):

$$
\frac{d}{d S}\left(\begin{array}{l}
\mathbf{X} \\
\mathbf{T} \\
\mathbf{G}
\end{array}\right)=\left(\begin{array}{lll}
0 & 1 & 0 \\
-\sigma \eta & 0 & \Sigma \\
0 & \sigma \Sigma & 0
\end{array}\right)\left(\begin{array}{l}
\mathbf{X} \\
\mathbf{T} \\
\mathbf{G}
\end{array}\right)
$$

where $\Sigma=\Sigma(S)$ is the dual geodesic curvature function of $\mathbf{X}(S) \in \mathbb{H}_{+}^{2}$ or $\mathbb{S}_{1}^{2}$.

Under the assumption that $\Sigma \neq \pm 1$, we define the dual evolute of $\mathbf{X}(S) \in \mathbb{H}_{+}^{2}$ or $\mathbb{S}_{1}^{2}$ as follows:

$$
\mathbf{B}(S)=\frac{\Sigma \mathbf{X}+\mathbf{G}}{\sqrt{\left|\Sigma^{2}-1\right|}} .
$$

We remark that $\mathbf{B}(S)$ is located in $\mathbb{H}_{+}^{2}$ if and only if $\Sigma^{2}>1$, otherwise it is in $\mathbb{S}_{1}^{2}$. Therefore, we consider a pseudo dual circle on $\mathbb{H}_{+}^{2}$ or $\mathbb{S}_{1}^{2}$ is described by the equation

$$
\mathbb{S}\left(R, \mathbf{B}_{0}\right)=\left\{\mathbf{X}(S) \in \mathbb{H}_{+}^{2} \text { or } \mathbb{S}_{1}^{2} \mid<\mathbf{X}, \mathbf{B}_{0}>=R(S)\right\}
$$

where $R=\rho+\varepsilon \rho^{*}$ is a dual spherical radius of curvature, and $\mathbf{B}_{0}$ is a fixed dual unit vector which determines the pseudo dual circle's center. Then, we have the following proposition.

Proposition 1. Let $\mathbf{X}: I \subseteq \mathbb{D} \rightarrow \mathbb{H}_{+}^{2}$ or $\mathbb{S}_{1}^{2}$ be a unit speed dual curve with $\Sigma^{2} \neq \pm 1$. Then $\frac{d \Sigma}{d S}=0$ iff $\mathbf{B}_{0}= \pm \mathbf{B}$. Under this condition, $\mathbf{X}(S) \in \mathbb{H}_{+}^{2}$ or $\mathbb{S}_{1}^{2}$ is a part of pseudo dual circle whose center is $\mathbf{B}$. Proof. For the first differential of $\mathbf{B}$ we get:

$$
\frac{d \mathbf{B}}{d S}=\mp \frac{\boldsymbol{\Sigma}^{\prime}}{\left|<\mathbf{X}^{\prime}, \mathbf{X}^{\prime}>\right|\left(\sqrt{\left|\Sigma^{2}-1\right|}\right)^{\frac{3}{2}}}\left(\boldsymbol{\Sigma}^{\prime} \mathbf{X}+\boldsymbol{\Sigma} \mathbf{G}\right) .
$$

Then $\mathbf{B}_{0}= \pm \mathbf{B}$ iff $\Sigma^{\prime}(S)=0$. Under this condition we put $R=\frac{\Sigma}{\sqrt{\left|\Sigma^{2}-1\right|}}$ with $\Sigma^{2} \neq \pm 1$. So $\mathbf{X}(S) \in \mathbb{H}_{+}^{2}$ or $\mathbb{S}_{1}^{2}$ is a part of pseudo dual circle whose center is $\mathbf{B}$.

Through the reminder of this work we will study a non-developable timelike ruled surface characterized by $(\sigma, \eta)=(-1,1)$. Therefore, we have

$$
\mathbf{X} \times \mathbf{T}=\mathbf{G}, \quad \mathbf{X} \times \mathbf{G}=-\mathbf{T}, \quad \mathbf{T} \times \mathbf{G}=-\mathbf{X}, \quad<\mathbf{G}, \mathbf{G}>=1,
$$

and under the assumption that $\Sigma^{2}>1$, we also have:

$$
\mathbf{B}(S)=\frac{\Sigma \mathbf{X}+\mathbf{G}}{\sqrt{\Sigma^{2}-1}} \text {, with }<\mathbf{B}, \mathbf{B}>=-1 .
$$


In terms of the Blaschke frame, we can show that:

$$
\begin{aligned}
d S & =\sqrt{<\mathbf{X}^{\prime}, \mathbf{X}^{\prime}>} d s \\
& =\sqrt{<\mathbf{x}^{\prime}, \mathbf{x}^{\prime}>+2 \varepsilon<\mathbf{x}^{\prime}, \mathbf{x}^{*^{\prime}}>d s} \\
& =\sqrt{1+2 \varepsilon<\mathbf{t}, \mathbf{C}^{\prime} \times \mathbf{x}+\mathbf{C} \times \mathbf{t}>} d s \\
& =(1+\varepsilon \mu) d s,
\end{aligned}
$$

which imply

$$
\frac{d s}{d S}=1-\varepsilon \mu
$$

Then, we determine $\Sigma=\Sigma(S)$ by

$$
\frac{d \mathbf{G}}{d S}=(1-\varepsilon \mu)\left\{\frac{d \mathbf{g}}{d s}+\varepsilon\left[\frac{d \mathbf{C}}{d s} \times \mathbf{g}+\mathbf{C} \times \frac{d \mathbf{g}}{d s}\right]\right\}
$$

This expression is further expanded using Eqs. (2.5) and (2.6) to yield

$$
\begin{aligned}
\frac{d \mathbf{G}}{d S} & =(1-\varepsilon \mu)\{-\gamma \mathbf{t}+\varepsilon[(-\Gamma \mathbf{x}+\mu \mathbf{g}) \times \mathbf{g}-\gamma \mathbf{C} \times \mathbf{t}]\} \\
& =(1-\varepsilon \mu)\left[-\gamma \mathbf{t}+\varepsilon\left(\Gamma \mathbf{t}-\gamma \mathbf{t}^{*}\right)\right] \\
& =(1-\varepsilon \mu)(-\gamma+\varepsilon \Gamma) \mathbf{T} \\
& =[-\gamma+\varepsilon(\Gamma+\gamma \mu)] \mathbf{T} .
\end{aligned}
$$

Comparing Eqs. (3.4) and (3.12) we see that $\Sigma$ is defined in terms of $\gamma, \mu$ and $\Gamma$ as:

$$
\Sigma=\gamma-\varepsilon(\Gamma+\gamma \mu) \text {. }
$$

Similar to the books in [14, 15], a dual point $\mathbf{B}_{0}$ of $\mathbb{H}_{+}^{2}$ will be said to be a $\mathbf{B}_{k}$ evolute of the dual curve $\mathbf{X}(S)$ in $\mathbb{H}_{+}^{2}$ at $S \in \mathbb{R}$ if, for all $i$ such that $1 \leq i \leq k,<\mathbf{B}_{0}, \mathbf{X}^{i}(S)>=0$, but $<\mathbf{B}_{0}, \mathbf{X}^{k+1}(S)>\neq$ 0 . Here $\mathbf{X}^{i}$ denotes the $\mathrm{i}$-th derivatives of $\mathbf{X}$ with respect to the dual arc length of $\mathbf{X}(S)$ in $\mathbb{H}_{+}^{2}$. For the first evolute $\mathbf{B}$ of $\mathbf{X}(S)$, we have $\left.\left\langle\mathbf{B}, \mathbf{X}^{\prime}\right\rangle= \pm<\mathbf{B}, \mathbf{T}\right\rangle=0$, and $\left.\left\langle\mathbf{B}, \mathbf{X}^{\prime \prime}\right\rangle= \pm<\mathbf{B}, \mathbf{X}+\Sigma \mathbf{G}\right\rangle=0$. So, $\mathbf{B}$ is at least a $\mathbf{B}_{2}$ evolute of $\mathbf{X}(S) \in \mathbb{H}_{+}^{2}$.

3.1. Height dual functions. Let $\mathbf{X}: I \subseteq \mathbb{D} \rightarrow \mathbb{H}_{+}^{2}$ be a dual curve $\mathbf{X}(S)$ in $\mathbb{H}_{+}^{2}$ with $\Sigma^{2}>1$. We now define a smooth dual function $H^{T}: I \times \mathbb{H}_{+}^{2} \rightarrow \mathbb{D}$, by $H^{T}\left(S, \mathbf{B}_{0}\right)=<\mathbf{B}_{0}, \mathbf{X}>$. We call $H^{T}$ a hyperbolic timelike height dual function on $\mathbf{X}(S)$ in $\mathbb{H}_{+}^{2}$. We use the notation $h_{\mathbf{e}}(S)=H^{T}\left(S, \mathbf{B}_{0}\right)$ for any fixed $\mathbf{B}_{0}$ of $\mathbb{H}_{+}^{2}$.

Proposition 2. Let $\mathbf{X}: I \subseteq \mathbb{D} \rightarrow \mathbb{H}_{+}^{2}$ be a dual curve $\mathbf{X}(S)$ in $\mathbb{H}_{+}^{2}$ with $\Sigma^{2}>1$. Then the following holds:

1- $h_{\mathbf{e}}$ will be invariant in the first approximation iff $\mathbf{B}_{0} \in S p\{\mathbf{X}, \mathbf{G}\}$, that is,

$$
h_{\mathbf{e}}^{\prime}=0 \Leftrightarrow<\mathbf{X}^{\prime}, \mathbf{B}_{0}>=0 \Leftrightarrow<\mathbf{T}, \mathbf{B}_{0}>=0 \Leftrightarrow \mathbf{B}_{0}=A_{1} \mathbf{X}+A_{2} \mathbf{G} ;
$$

for some dual numbers $A_{1}, A_{2} \in \mathbb{D}$, and $A_{1}^{2}-A_{2}^{2}=-1$.

2- $h_{\mathbf{e}}$ will be invariant in the second approximation iff $\mathbf{B}_{0}$ is $\mathbf{B}_{2}$ evolute of $\mathbf{X}(S) \in \mathbb{H}_{+}^{2}$, that is,

$$
h_{\mathbf{e}}^{\prime}=h_{\mathbf{e}}^{\prime \prime}=0 \Leftrightarrow \mathbf{B}_{0}= \pm \mathbf{B} \text {, and } \Sigma^{2}>1 .
$$

3- $h_{\mathbf{e}}$ will be invariant in the third approximation iff $\mathbf{B}_{0}$ is $\mathbf{B}_{3}$ evolute of $\mathbf{X}(S) \in \mathbb{H}_{+}^{2}$, that is,

$$
h_{\mathbf{e}}^{\prime}=h_{\mathbf{e}}^{\prime \prime}=h_{\mathbf{e}}^{\prime \prime \prime}=0 \Leftrightarrow \mathbf{B}_{0}= \pm \mathbf{B}, \Sigma^{2}>1 \text {, and } \Sigma^{\prime} \neq 0 .
$$

4- $h_{\mathbf{e}}$ will be invariant in the fourth approximation iff $\mathbf{B}_{0}$ is $\mathbf{B}_{4}$ evolute of $\mathbf{X}(S) \in \mathbb{H}_{+}^{2}$, that is,

$$
h_{\mathbf{e}}^{\prime}=h_{\mathbf{e}}^{\prime \prime}=h_{\mathbf{e}}^{\prime \prime \prime}=h_{\mathbf{e}}^{(i v)}=0 \Leftrightarrow \mathbf{B}_{0}= \pm \mathbf{B}, \Sigma^{2}>1, \Sigma^{\prime}=0 \text {, and } \Sigma^{\prime \prime} \neq 0 .
$$

Proof. For the first differential of $h_{\mathbf{e}}$ we get:

$$
h_{\mathbf{e}}^{\prime}=<\mathbf{X}^{\prime}, \mathbf{B}_{0}>\text {. }
$$

So, we get:

$$
h_{\mathbf{e}}^{\prime}=0 \Leftrightarrow<\mathbf{T}, \mathbf{B}_{0}>=0 \Leftrightarrow \mathbf{B}_{0}=A_{1} \mathbf{X}+A_{2} \mathbf{G}
$$


for some dual numbers $A_{1}, A_{2} \in \mathbb{D}$, and $A_{1}^{2}-A_{2}^{2}=-1$, the result is clear.

2- Differentiation of Eq. (3.18) leads to:

$$
h_{\mathbf{e}}^{\prime \prime}=<\mathbf{X}^{\prime \prime}, \mathbf{B}_{0}>=<\mathbf{X}+\Sigma \mathbf{G}, \mathbf{B}_{0}>\text {. }
$$

By using Eq. (3.19),we have:

$$
h_{\mathbf{e}}^{\prime}=h_{\mathbf{e}}^{\prime \prime}=0 \Leftrightarrow<\mathbf{X}^{\prime}, \mathbf{B}_{0}>=<\mathbf{X}^{\prime \prime}, \mathbf{B}_{0}>=0 \Leftrightarrow \mathbf{B}_{0}= \pm \frac{\mathbf{X}^{\prime} \times \mathbf{X}^{\prime \prime}}{\left\|\mathbf{X}^{\prime} \times \mathbf{X}^{\prime \prime}\right\|}= \pm \mathbf{B} .
$$

3- Differentiation of Eq. (3.20) leads to:

$$
h_{\mathbf{e}}^{\prime \prime \prime}=<\mathbf{X}^{\prime \prime \prime}, \mathbf{B}_{\mathbf{0}}>=\left(1-\Sigma^{2}\right)<\mathbf{T}, \mathbf{B}_{0}>+\boldsymbol{\Sigma}^{\prime}<\mathbf{G}, \mathbf{B}_{0}>
$$

Hence, we have:

$$
h_{\mathbf{e}}^{\prime}=h_{\mathbf{e}}^{\prime \prime}=h_{\mathbf{e}}^{\prime \prime \prime}=0 \Leftrightarrow \mathbf{B}_{0}= \pm \mathbf{B}, \Sigma^{2}>1, \text { and } \Sigma^{\prime} \neq 0 .
$$

4- By the similar arguments, we can also have:

$$
h_{\mathbf{e}}^{\prime}=h_{\mathbf{e}}^{\prime \prime}=h_{\mathbf{e}}^{\prime \prime \prime}=h_{\mathbf{e}}^{(i v)}=0 \Leftrightarrow \mathbf{B}_{0}= \pm \mathbf{B}, \Sigma^{2}>1, \Sigma^{\prime}=0 \text {, and } \Sigma^{\prime \prime} \neq 0 .
$$

The proof is completed.

According to the above proposition, we have:

(a) The osculating circle $\mathbb{S}\left(R, \mathbf{B}_{0}\right)$ of $\mathbf{X}(S)$ in $\mathbb{H}_{+}^{2}$ is determined by the equations

$$
<\mathbf{B}_{0}, \mathbf{X}>=R,<\mathbf{X}^{\prime}, \mathbf{B}_{0}>=0,<\mathbf{X}^{\prime \prime}, \mathbf{B}_{0}>=0,
$$

which are obtained from the condition that the osculating circle should have contact of at least third order at $\mathbf{X}\left(S_{0}\right)$ iff $\Sigma^{\prime} \neq 0$. Then, as in the Euclidean 3-space, the first and last two equations, respectively, determine the osculating timelike line congruence of the trajectory of the line $\mathbf{X}$ and its axis $\mathbf{B}_{0}[12]$.

(b) The osculating circle $\mathbb{S}\left(R, \mathbf{B}_{0}\right)$ and the dual curve $\mathbf{X}(S)$ in $\mathbb{H}_{+}^{2}$ have at least fourth order at $\mathbf{X}\left(S_{0}\right)$ iff $\Sigma^{\prime}=0$, and $\Sigma^{\prime \prime} \neq 0$.

In this way, considering the evolutes of a general timelike ruled surface we can get a sequence of evolutes $\mathbf{B}_{2}, \mathbf{B}_{3}, \ldots, \mathbf{B}_{n}$. The properties and the relationship between these evolutes and their involute timelike surfaces are very interesting problems. For example, it is easy to see that when $\mathbf{B}_{0}= \pm \mathbf{B}$, and $\Sigma^{\prime} \neq 0, M$ is traced during a Lorentzian screw motion about $\mathbf{B}_{0}$, by the line $\mathbf{X}$ located at $R=$ const. relative to $\mathbf{B}_{0}$.

\section{UnFOLDINGS OF DUAL FUNCTIONS OF ONE VARIABLE}

In this section we will use the same technique on the singularity theory for families of dual smooth functions. Detailed descriptions are found in the books $[11,12]$. Let $F:\left(\mathbb{D} \times \mathbb{D}^{r},\left(S_{0}, \mathbf{X}_{0}\right)\right) \rightarrow \mathbb{D}$ be a dual smooth function, and $\mathcal{F}(S)=F_{X_{0}}, F_{X_{0}}(S)=F\left(S, \mathbf{X}_{0}\right)$. Then $F$ is called an r-parameter dual unfolding of $\mathcal{F}(S)$. We say that $\mathcal{F}(S)$ has $A_{k}$ singularity at $S_{0}$ if $\mathcal{F}^{(p)}\left(S_{0}\right)=0$ for all $1 \leq p \leq k$, and $\mathcal{F}^{(p+1)}\left(S_{0}\right) \neq 0$. We also say that $\mathcal{F}(S)$ has $A_{\geq k}$ singularity at $S_{0}$ if $\mathcal{F}^{(p)}\left(S_{0}\right)=0$ for all $1 \leq p \leq k$. Let the $(k-1)$-jet of the partial derivative $\frac{\partial F}{\partial X_{i}}$ at $S_{0}$ be $j^{(k-1)}\left(\frac{\partial F}{\partial X_{i}}\left(S, \mathbf{X}_{0}\right)\right)=\Sigma_{j=1}^{k-1} L_{j i} S^{j}$ (without the dual constant term), for $i=1, \ldots, r$. Then $F(S, \mathbf{X})$ is called a $(p)$ versal dual unfolding iff the $(k-1) \times r$ dual matrix of coefficients $\left(L_{j i}\right)$ has rank $(k-1)$. (This certainly requires $k-1 \leq r$, so the smallest value of $r$ is $k-1$ ).

We now state important sets about the unfoldings relative to the above notations. The singular dual set of $F(S, \mathbf{X})$ is the set

$$
\mathbb{S}_{F}=\left\{\mathbf{X} \in \mathbb{H}_{+}^{2} \mid \text { there exists } S \text { with } \frac{\partial F}{\partial S}=0 \text { at }(S, \mathbf{X})\right\} .
$$

The bifurcation dual set $\mathbb{B}_{F}$ of $F$ is the set $[11,12]$ :

$$
\mathbb{B}_{F}=\left\{\mathbf{X} \in \mathbb{H}_{+}^{2} \mid \text { there exists } S \text { with } \frac{\partial F}{\partial S}=\frac{\partial^{2} F}{\partial S^{2}}=0 \text { at }(S, \mathbf{X})\right\} .
$$


Then similar to [11], we state the following theorem:

Theorem 1. Let $F: \mathbb{D} \times \mathbb{D}^{r},\left(\left(S_{0}, \mathbf{X}_{0}\right)\right) \rightarrow \mathbb{D}$ be a dual r-parameter unfolding of $\mathcal{F}(S)$, which has the $A_{k}$ singularity $(k \geq 1)$ at $S_{0}$. Suppose that $F$ is a $(p)$ versal dual unfolding Then:

(1) If $k=2$, then $\mathbb{B}_{F}$ is locally diffepmorphic to $\{\mathbf{0}\} \times \mathbb{D}^{r-1}$;

(2) If $k=3$, then $\mathbb{B}_{F}$ is locally diffepmorphic to $\widetilde{\mathbf{C}} \times \mathbb{D}^{r-2}$, where $\widetilde{\mathbf{C}}=\left\{\left(X_{1}, X_{2}\right) \mid X_{1}^{2}=X_{2}^{3}\right\}$ is the ordinary cusp.

For the dual curve $\mathbf{X}(S) \in \mathbb{H}_{+}^{2}$, with $\Sigma^{2}>1$, and $h_{\mathbf{e}}(S)=H^{T}\left(S, \mathbf{B}_{0}\right)$, the bifurcation dual set of $H^{T}$ is given as follows:

$$
\mathbb{B}_{H^{T}}=\left\{\mathbf{X} \in \mathbb{H}_{+}^{2} \mid \mathbf{B}= \pm \frac{\Sigma \mathbf{X}+\mathbf{G}}{\sqrt{\Sigma^{2}-1}}, \Sigma^{2}>1\right\} .
$$

Hence, we have the following fundamental proposition:

Proposition 3. For the dual unit speed curve $\mathbf{X}(S)=\left(X_{1}(S), X_{2}(S), X_{3}(S)\right)$ on $\mathbb{H}_{+}^{2}$, with $\Sigma\left(S_{0}\right) \neq 0$ and $\Sigma^{2}\left(S_{0}\right) \neq \pm 1$. If the $h_{\mathbf{e}}(S)=H^{T}\left(S, \mathbf{B}_{0}\right)$ has the $A_{k}$-singularity $(k=2,3)$ at $S_{0} \in \mathbb{D}$, then $H^{T}$ is the $(p)$ versal dual unfolding of $h_{\mathbf{e}_{0}}\left(S_{0}\right)$.

Proof. Since $\mathbf{B}_{0}=\left(Z_{1}, Z_{2}, Z_{3}\right) \in \mathbb{H}_{+}^{2},-Z_{1}^{2}+Z_{2}^{2}+Z_{3}^{2}=-1, Z_{1}>0 . Z_{1}, Z_{2}$, and $Z_{3}$ can't be all zero. Without loss of generality, suppose $Z_{3} \neq 0$. Then by $Z_{3}=\sqrt{-1+Z_{1}^{2}-Z_{2}^{2}}$, we have

$$
H^{T}\left(S, \mathbf{B}_{0}\right)=-Z_{1} X_{1}(S)+Z_{2} X_{2}(S)+\sqrt{-1+Z_{1}^{2}-Z_{2}^{2}} X_{3}(S) .
$$

So

We also have

$$
\left.\begin{array}{l}
\frac{\partial H^{T}}{\partial Z_{1}}=\left(-X_{1}(S)+\frac{Z_{1} X_{3}(S)}{\sqrt{-1+Z_{1}^{2}-Z_{2}^{2}}}\right), \\
\frac{\partial H^{T}}{\partial Z_{2}}=\left(X_{2}(S)-\frac{Z_{2} X_{3}(S)}{\sqrt{-1+Z_{1}^{2}-Z_{2}^{2}}}\right) .
\end{array}\right\}
$$

and

$$
\left.\begin{array}{rl}
\frac{\partial}{\partial S} \frac{\partial H^{T}}{\partial Z_{1}} & =\left(-X_{1}^{\prime}(S)+\frac{Z_{1} X_{3}^{\prime}(S)}{\sqrt{-1+Z_{1}^{2}-Z_{2}^{2}}}\right), \\
\frac{\partial}{\partial S} \frac{\partial H^{T}}{\partial Z_{2}} & =\left(X_{2}^{\prime}(S)-\frac{Z_{2} X_{3}^{\prime}(S)}{\sqrt{-1+Z_{1}^{2}-Z_{2}^{2}}}\right),
\end{array}\right\}
$$

$$
\left.\begin{array}{rl}
\frac{\partial^{2}}{\partial S^{2}} \frac{\partial H^{T}}{\partial Z_{1}} & =\left(-X_{1}^{\prime \prime}(S)+\frac{Z_{1} X_{3}^{\prime \prime}(S)}{\sqrt{-1+Z_{1}^{2}-Z_{2}^{2}}}\right), \\
\frac{\partial^{2}}{\partial S^{2}} \frac{\partial H^{T}}{\partial Z_{2}} & =\left(X_{2}^{\prime \prime}(S)-\frac{Z_{2} X_{3}^{\prime \prime}(S)}{\sqrt{-1+Z_{1}^{2}-Z_{2}^{2}}}\right) \cdot
\end{array}\right\}
$$

Let $\mathbf{B}_{0}=\left(Z_{10}, Z_{20}, Z_{30}\right) \in \mathbb{H}_{+}^{2}$, and assume $Z_{30} \neq 0$, then

$$
\left.\begin{array}{l}
j^{1}\left(\frac{\partial H^{T}}{\partial Z_{1}}\left(S, \mathbf{B}_{0}\right)\right)=\left(-X_{1}^{\prime}\left(S_{0}\right)+\frac{Z_{1} X_{3}^{\prime}\left(S_{0}\right)}{Z_{30}}\right) S, \\
j^{1}\left(\frac{\partial H^{T}}{\partial Z_{2}}\left(S, \mathbf{B}_{0}\right)\right)=\left(X_{2}^{\prime}\left(S_{0}\right)-\frac{Z_{2} X_{3}^{\prime}\left(S_{0}\right)}{Z_{30}}\right) S,
\end{array}\right\}
$$

and

$$
\begin{gathered}
j^{2}\left(\frac{\partial H^{T}}{\partial Z_{1}}\left(S, \mathbf{B}_{0}\right)\right)=\left(-X_{1}^{\prime}\left(S_{0}\right)+\frac{Z_{1} X_{3}^{\prime}\left(S_{0}\right)}{Z_{30}}\right) S \\
+\frac{1}{2}\left(-X_{1}^{\prime \prime}\left(S_{0}\right)+\frac{Z_{1} X_{3}^{\prime \prime}\left(S_{0}\right)}{Z_{30}}\right) S^{2} \\
j^{2}\left(\frac{\partial H^{T}}{\partial Z_{2}}\left(S, \mathbf{B}_{0}\right)\right)=\left(X_{2}^{\prime}\left(S_{0}\right)-\frac{Z_{2} X_{3}^{\prime}\left(S_{0}\right)}{Z_{30}}\right) S \\
+\frac{1}{2}\left(X_{2}^{\prime \prime}\left(S_{0}\right)-\frac{Z_{2} X_{3}^{\prime \prime}\left(S_{0}\right)}{Z_{30}}\right) S^{2} .
\end{gathered}
$$

(i) If $h_{\mathbf{e}_{0}}\left(S_{0}\right)$ has the $A_{2}$-singularity at $S_{0} \in \mathbb{D}$, then $h_{\mathbf{e}_{0}}^{\prime}\left(S_{0}\right)=0$. So the $(2-1) \times 2$ dual matrix of coefficients $\left(L_{j i}\right)$ is:

$$
A=\left(-X_{1}^{\prime}\left(S_{0}\right)+\frac{Z_{1} X_{3}^{\prime}\left(S_{0}\right)}{Z_{30}} \quad X_{2}^{\prime}\left(S_{0}\right)-\frac{Z_{2} X_{3}^{\prime}\left(S_{0}\right)}{Z_{30}}\right) ;
$$


Suppose that the rank of the matrix $A$ is zero, then we have:

$$
X_{1}^{\prime}\left(S_{0}\right)=\frac{Z_{1} X_{3}^{\prime}\left(S_{0}\right)}{Z_{30}}, X_{2}^{\prime}\left(S_{0}\right)=\frac{Z_{2} X_{3}^{\prime}\left(S_{0}\right)}{Z_{30}} .
$$

Since $\left\|\mathbf{X}^{\prime}\left(S_{0}\right)\right\|=\left\|\mathbf{T}\left(S_{0}\right)\right\|=1$, we have $X_{3}^{\prime}\left(S_{0}\right) \neq 0$, so that we have the contradiction as follows:

$$
\begin{aligned}
0 & =<\left(X_{1}^{\prime}\left(S_{0}\right), X_{2}^{\prime}\left(S_{0}\right), X_{3}^{\prime}\left(S_{0}\right)\right),\left(Z_{1}, Z_{2}, Z_{30}\right)> \\
& =-X_{1}^{\prime}\left(S_{0}\right) Z_{1}+X_{2}^{\prime}\left(S_{0}\right) Z_{2}+X_{3}^{\prime}\left(S_{0}\right) Z_{30} \\
& =-\frac{Z_{1}^{2} X_{3}^{\prime}\left(S_{0}\right)}{Z_{30}}+\frac{Z_{2}^{2} X_{3}^{\prime}\left(S_{0}\right)}{Z_{30}}+X_{3}^{\prime}\left(S_{0}\right) Z_{30} \\
& =\frac{-X_{3}^{\prime}\left(S_{0}\right)}{Z_{30}} \neq 0 .
\end{aligned}
$$

Therefore rank $(A)=1$, and $H^{T}$ is the (p) versal dual unfolding of $h_{\mathbf{e}}$ at $S_{0}$.

(ii) If $h_{\mathbf{e}_{0}}\left(S_{0}\right)$ has the $A_{3}$-singularity at $S_{0} \in \mathbb{D}$, then $h_{\mathbf{e}_{0}}^{\prime}\left(S_{0}\right)=h_{\mathbf{e}_{0}}^{\prime \prime}\left(S_{0}\right)=0$, and by Proposition 1 :

$$
\mathbf{B}\left(S_{0}\right)= \pm\left(\frac{\Sigma \mathbf{X}+\mathbf{G}}{\sqrt{\Sigma^{2}-1}}\right)\left(S_{0}\right)
$$

where $\Sigma^{2}\left(S_{0}\right) \neq \pm 1, \Sigma^{\prime}\left(S_{0}\right)=0$, and $\Sigma^{\prime \prime}\left(S_{0}\right) \neq 0$. So the $(3-1) \times 2$ dual matrix of the coefficients $\left(L_{j i}\right)$ is

$$
B=\left(\begin{array}{ll}
L_{11} & L_{12} \\
L_{21} & L_{22}
\end{array}\right)=\left(\begin{array}{cc}
-X_{1}^{\prime}+\frac{Z_{1} X_{3}^{\prime}}{Z_{30}} & X_{2}^{\prime}-\frac{Z_{2} X_{3}^{\prime}}{\sqrt{-1+Z_{1}^{2}-Z_{2}^{2}}} \\
-X_{1}^{\prime \prime}+\frac{Z_{1} X_{3}^{\prime \prime}}{Z_{30}} & X_{2}^{\prime \prime}-\frac{Z_{2} X_{3}^{\prime \prime}}{Z_{30}}
\end{array}\right)
$$

For the purpose, we also require the $2 \times 2$ matrix $B$ to be non-singular, which always does. In fact, the determinate of this matrix at $S_{0}$ is

$$
\begin{aligned}
\operatorname{det}(B) & =\frac{1}{Z_{30}}\left|\begin{array}{ccc}
-X_{1}^{\prime} & X_{2}^{\prime} & X_{3}^{\prime} \\
-X_{1}^{\prime \prime} & X_{2}^{\prime \prime} & X_{3}^{\prime \prime} \\
Z_{10} & Z_{20} & Z_{30}
\end{array}\right| \\
& =\frac{1}{Z_{30}}<\mathbf{X}^{\prime} \times \mathbf{X}^{\prime \prime}, \mathbf{E}_{\mathbf{0}}> \\
& = \pm \frac{1}{Z_{30}}<\mathbf{X}^{\prime} \times \mathbf{X}^{\prime \prime},\left(\frac{\Sigma \mathbf{X}+\mathbf{G}}{\sqrt{\Sigma^{2}-1}}\right)>
\end{aligned}
$$

Since $\mathbf{X}^{\prime}=\mathbf{T}$, we have $\mathbf{X}^{\prime \prime}=\mathbf{X}+\Sigma \mathbf{G}$. Substituting these relations to the above equality, we have

$$
\operatorname{det}(B)=\mp \frac{1}{Z_{30}} \frac{\Sigma\left(S_{0}\right)}{\sqrt{\Sigma^{2}\left(S_{0}\right)-1}} \neq 0 .
$$

This means that $\operatorname{rank}(B)=2$. This completes the proof.

Theorem 2. Let $\mathbf{X}(S)$ be a dual unit speed curve on $\mathbb{H}_{+}^{2}$, then the dual spherical evolute of $\mathbf{X}(S)$ is: (1) Diffepmorphic to a timelike oriented line if $\Sigma^{\prime}\left(S_{0}\right) \neq 0$;

(2) Diffepmorphic to the cusp $\widetilde{\mathbf{C}}$ at $S_{0} \in \mathbb{D}$ if $\Sigma^{\prime}\left(S_{0}\right)=0$, and $\Sigma^{\prime \prime}\left(S_{0}\right) \neq 0$.

Proof. For the proof of assertion (1), from Eq. (3.9) we have

$$
\frac{d \mathbf{B}}{d S}:=\mathbf{B}^{\prime}=\mp \frac{\boldsymbol{\Sigma}^{\prime}}{\left(\sqrt{\Sigma^{2}-1}\right)^{\frac{3}{2}}}\left(\boldsymbol{\Sigma}^{\prime} \mathbf{X}+\mathbf{\Sigma} \mathbf{G}\right) .
$$

Therefore $\mathbf{B}$ is locally diffepmorphic to a timelike oriented line if $\Sigma^{\prime}\left(S_{0}\right) \neq 0$. For the assertion (2), from Proposition 2, and Theorem 1, the bifurcation set $\mathbb{B}_{H^{T}}$ at $\mathbf{B}_{0}= \pm\left(\frac{\Sigma \mathbf{X}+\mathbf{G}}{\sqrt{\Sigma^{2}-1}}\right)\left(S_{0}\right)$ is locally diffepmorphic to the ordinary cusp $\widetilde{\mathbf{C}}$ in $\mathbb{H}_{+}^{2}$ if $\Sigma^{\prime}\left(S_{0}\right)=0$, and $\Sigma^{\prime \prime}\left(S_{0}\right) \neq 0$. 
Example 1. The dual coordinates $X_{i}=\left(x_{i}+\varepsilon x_{i}^{*}\right)$ of an arbitrary point $\mathbf{X}$ of the dual hyperbolic unit sphere $\mathbb{H}_{+}^{2}$, centered at the origin, may be expressed as:

$$
M: \mathbf{X}=(\cosh \Theta, \sinh \Theta \cos \Phi, \sinh \Theta \sin \Phi),
$$

where $\Theta=\vartheta+\varepsilon \vartheta^{*}$, and $\Phi=\varphi+\varepsilon \varphi^{*}$ are dual hyperbolic and spacelike angles with $\vartheta^{*}, \vartheta \in \mathbb{R}$, and $0 \leq \varphi \leq 2 \pi$, respectively. Moreover, let us consider $\mathbf{X}=\mathbf{X}(t), t \in \mathbb{R}$ corresponding to a timelike ruled surface $M$.Therefore, we have:

$$
\left(\begin{array}{l}
\mathbf{X} \\
\mathbf{T} \\
\mathbf{G}
\end{array}\right)=\left(\begin{array}{lll}
\cosh \Theta & \sinh \Theta \cos \Phi & \sinh \Theta \sin \Phi \\
0 & -\sin \Phi & \cos \Phi \\
\sinh \Theta & \cosh \Theta \cos \Phi & \cosh \Theta \sin \Phi
\end{array}\right)\left(\begin{array}{l}
\mathbf{F}_{1} \\
\mathbf{F}_{2} \\
\mathbf{F}_{3}
\end{array}\right) .
$$

Thus, we get the Blaschke equations:

$$
\frac{d}{d t}\left(\begin{array}{l}
\mathbf{X} \\
\mathbf{T} \\
\mathbf{G}
\end{array}\right)=\left(\begin{array}{ccc}
0 & \frac{d \Phi}{d t} \sinh \Theta & \frac{d \Theta}{d t} \\
\frac{d \Phi}{d t} \sinh \Theta & 0 & -\frac{d \Phi}{d t} \cosh \Theta \\
\frac{d \Theta}{d t} & \frac{d \Phi}{d t} \cosh \Theta & 0
\end{array}\right)\left(\begin{array}{c}
\mathbf{X} \\
\mathbf{T} \\
\mathbf{G}
\end{array}\right),
$$

from which we obtain

$$
\left.\begin{array}{l}
d S=\sqrt{\left(\frac{d \Phi}{d t} \sinh \Theta\right)^{2}+\left(\frac{d \Theta}{d t}\right)^{2}} d t \\
\Sigma(t)=\frac{\frac{d \Phi}{d t} \cosh \Theta}{\sqrt{\left(\frac{d \Phi}{d t} \sinh \Theta\right)^{2}+\left(\frac{d \Theta}{d t}\right)^{2}}} .
\end{array}\right\}
$$

Now, let us take $\vartheta=c_{1}$ (real const.), and $\vartheta^{*}=c_{2}$ (real const.). In this case, we have

$$
\left.\begin{array}{l}
\mu:=\frac{d s^{*}}{d s}=\left(\frac{d \varphi^{*}}{d t} / \frac{d \varphi}{d t}\right)+\vartheta^{*} \operatorname{coth} \vartheta, \\
\gamma=\operatorname{coth} \vartheta,-\Gamma=\left(\frac{d \varphi^{*}}{d t} / \frac{d \varphi}{d t}\right) \operatorname{coth} \vartheta+\vartheta^{*},
\end{array}\right\}
$$

and the dual evolute $\mathbf{B}$ is given as follows:

$$
\mathbf{B}(t)=\frac{\Sigma \mathbf{X}-\mathbf{G}}{\sqrt{\Sigma^{2}-1}}=\mathbf{F}_{1} .
$$

According to Theorem 2, we have that the evolute of $\mathbf{X}=\mathbf{X}(t)$ in $\mathbb{H}_{+}^{2}$ is locally diffeomorphic to a timelike oriented line. Moreover, let $\mathbf{y}\left(y_{1}, y_{2}, y_{3}\right)$ denote the position vector of an arbitrary point of $M$. Then, considering Eqs. (3.1), and (4.19) we find:

$$
\mathbf{y}(\varphi, v)=\left(\begin{array}{c}
\varphi^{*}+v \cosh \vartheta \\
\vartheta^{*} \sin \varphi+v \sinh \vartheta \cos \varphi \\
-\vartheta^{*} \cos \varphi+v \sinh \vartheta \sin \varphi
\end{array}\right), v \in \mathbb{R} .
$$

From Eq. (4.25) we have:

$$
M: \frac{y_{2}^{2}}{\vartheta^{* 2}}+\frac{y_{3}^{2}}{\vartheta^{* 2}}-\frac{Y_{1}^{2}}{\left(\vartheta^{*} \operatorname{coth} \vartheta\right)^{2}}=1,
$$

where $Y_{1}=y_{1}-\varphi^{*}$. Since $\vartheta$, and $\vartheta^{*}$ are two-independent parameters, we can say that $M$ is, in generally, a family of Lorentzian one-sheeted hyperboloids with two parameters, so it represents a quadratic timelike line congruence. The intersection of this timelike congruence and the corresponding spacelike planes $y_{1}-\varphi^{*}=0$ is the one-parameter family of Euclidean circles $y_{2}^{2}+y_{3}^{2}=\vartheta^{* 2}$. If we take $\varphi^{*}(t)=h \varphi$, and $\varphi(t)=\varphi$ then we immediately obtain a member of this line congruence as shown in Fig. 2. ;

$$
\varphi \in[0,2 \pi], v \in[-3,3], \vartheta=\frac{\pi}{2}, \vartheta^{*}=h=1 .
$$

\section{Conclusion}

Mathematical techniques used the E. Study's map have been shown to be suitable for study dual hyperbolic invariants as applications of the singularity theory of smooth dual functions. Hopefully these results will lead to a wider usage of the geometric properties of the timelike ruled surfaces An analogue of the problem addressed in this paper may be consider for $\mathbf{X}(S) \in \mathbb{S}_{1}^{2}$ in the dual Lorentzian 3 -space $\mathbb{D}_{1}^{3}$. We will study this problem in the future. 


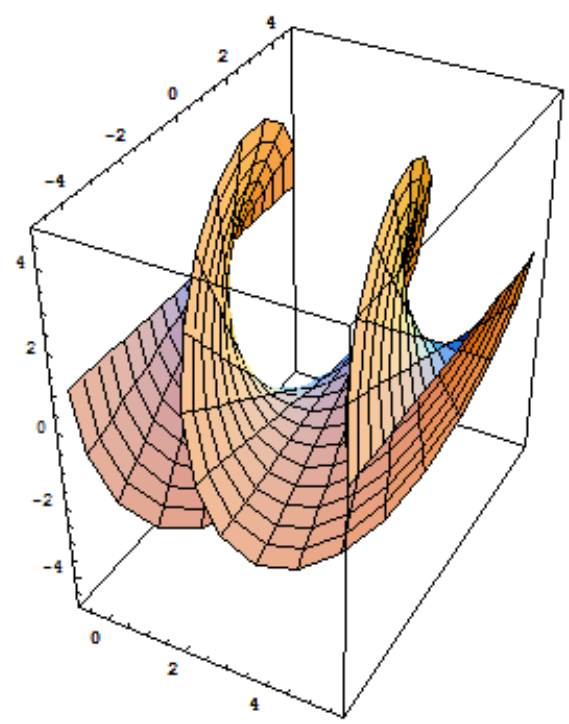

FIGURE 2

\section{REFERENCES}

[1] O. Bottema, and B. Roth. Theoretical Kinematics, North-Holland Press, New York 1979.

[2] A. Karger, and J. Novak. Space Kinematics and Lie Groups, Gordon and Breach Science Publishers, New York 1985.

[3] J M. MC Carthy J M. An introduction to theoretical kinematics, London: The MIT Press 1990.

[4] H. Pottman, and J. Wallner. Computational Line Geometry, Springer-Verlag, Berlin, Heidelberg 2001.

[5] R.A. Abdel-Baky, and F.R. Al-Solamy. A new geometrical approach to one-parameter spatial motion, J. Eng. Math. 60 (2008). 149-172.

[6] R.A. Abdel-Baky, and R.A Al-Ghefari. On the one-parameter dual spherical motions, Comput. Aided Geom. Des. 28 (2011), 23-37.

[7] R.A Al-Ghefari, and R.A. Abdel-Baky. Kinematic geometry of a line trajectory in spatial motion, J. Mech. Sci. Tech. 29 (9) (2015), 3597-3608.

[8] B. O’Neil. Semi-Riemannian Geometry geometry, with applications to relativity, Academic Press, New York, 1983.

[9] W. Sodsiri. Ruled linear Weingarten surfaces in Minkowski 3-space, Soochow J. Math., 29(4) (2003), 435-443.

[10] W. Kuhnel. Differential Geometry (2nd Edition), Amer. Math. Soc., 2006.

[11] R. Lopez. Differential Geometry of Curves and Surfaces in Lorentz-Minkowski Space, arxiv.org/abs/0810.3351v1 2008.

[12] Y. Yayli, A. Caliskan, and H. H. Ugurlu, The E. Study Maps of Circles on Dual Hyperbolic and Lorentzian Unit Spheres $\mathrm{H}_{0}^{2}$ and $\mathrm{S}_{0}^{2}$, Math. Proc. R. Ir. Acad. 102A (2002), no. 1, 37-47.

[13] M. Onder, and HH Ugurlu. Frenet frames and invariants of timelike ruled surfaces, Ain Shams Eng. J. 4 (2013), $507-513$.

[14] Bruce, J. W.; Giblin, P. J.: Curves and Singularities. 2nd. ed. Cambridge Univ. Press, Cambridge 1992.

[15] IR. Porteous. Geometric differentiation for the intelligence of Curves and Surfaces, Second edition, Cambridge University Press, Cambridge, 2001.

[16] S. Izumiya and N. Takeuchi, Geometry of ruled surfaces, Applicable Math., in the golden age, (2003), 305-338.

[17] S. Izumiya and N. Takeuchi. Special Curves and Ruled Surfaces, Beitrage zur Algebra und Geometrie Contributions to Algebra and Geometry, 44 (2003), No. 1, 203-212.

[18] S. Izumiya and A. Takiyama, A time-like surface in Minkowski 3-space which contains pseudocircles, Proc. Edinburgh Math. Soc. (2) 40, 1 (1997), 27-136.

[19] S. Izumiya, D-H. Pe. and T. Sano. The lightcone Gauss map and lightcone developable of a spacelike curve in Minkowski 3-space, J. Glasgow Math. J. 42 (2000), 75-89.

[20] S. Izumiya, D. Pei and T. Sano, Singularities of hyperbolic Gauss maps, Proc. London Math. Soc., 86 (2003), $485-512$.

[21] S. Izumiya and M. C. Romero Fuster, The horospherical Gauss-Bonnet type theorem in hyperbolic space, J. Math. Soc. Japan 58 (2006), 965-984.

[22] S. Izumiya, K. Saji and N. Takeuchi, Circular surfaces, Adv. Geom. 7 (2007), 295-313.

[23] S. Izumiya, Legendrian dualities and spacelike hypersurfaces in the lightcone, Mosc. Math. J. 9 (2009), 325-357.

${ }^{1}$ Department of Mathematics, Sciences Faculty for Girls, King Abdulaziz University, P.O. Box 126300, JedDAh 21352, SAUdi Arabia 
ABDEL-BAKY

${ }^{2}$ Department of Mathematics, Faculty of Science, University of Assiut, Assiut 71516, Egypt

*CORRESPONDING AUthor: rbaky@Live.com 